\title{
Adaptación y dimensionalidad de la escala DOCS en el contexto organizacional costarricense: aplicación de la entrevista cognitiva y el análisis factorial confirmatorio
}

\author{
Adaptation and Dimensionality of the DOCS Scale in the Costa Rican \\ Organizational Context: An Application of the Cognitive Interview and of \\ Confirmatory Factor Analysis
}

\author{
Tomás Vargas-Halabí \\ Universidad de Costa Rica, Costa Rica
}

\author{
Ronald Mora-Esquivel \\ Instituto Tecnológico de Costa Rica, Costa Rica
}

\begin{abstract}
Resumen
El presente estudio tiene como objetivo presentar la utilidad de la Entrevista Cognitiva como herramienta para ajustar, al contexto organizacional costarricense, la escala Denison (DOCS, por sus siglas en inglés) para medir la cultura organizacional previamente adaptada a Valencia, España, por Bonavia, Prado y Barberá (2009). La DOCS fue desarrollada, de forma original en inglés por Denison (1984), Denison \& Mishra (1995) y Fey \& Denison (2003). Para la adaptación, se aplicó la Entrevista Cognitiva a una muestra de 15 individuos con cargos de supervisión o gerencia en organizaciones con 25 o más empleados. Con base en los resultados de las entrevistas, se ajustó el lenguaje de la escala al contexto nacional. Posteriormente, esta última se aplicó en dos muestras de participantes con características similares, pero provenientes de dos poblaciones diferentes, concretamente: a) 314 estudiantes, de un total de 445, de las Maestrías en Administración de Empresas y de Gestión de Proyectos del Instituto Tecnológico de Costa Rica que, para su admisión, requieren experiencia profesional previa, preferiblemente en cargos de supervisión y gerencia; b) 368 gerentes de un marco muestral de 1743 empresas provenientes de diversas asociaciones empresariales nacionales. En ambas muestras se efectuaron 8 análisis factoriales confirmatorios utilizando el software EQS 6.3, con el fin de explorar la dimensionalidad de la escala adaptada y depurar la versión final. Los resultados confirman que la Entrevista Cognitiva fue una herramienta valiosa en este proceso y que la escala final (denominada DOCSCR-V2) muestra un adecuado funcionamiento en el contexto organizacional costarricense.
\end{abstract}

Palabras clave: Modelo DOCS, entrevista cognitiva, adaptación de escalas, cultura organizativa, SEM, análisis factorial confirmatorio

\begin{abstract}
:
The present study aims to present the Cognitive Interview as a useful pre-test tool for adapting the Denison Organizational Culture Survey (DOCS) to the Costa Rican business context, it was previously adapted in Valencia, Spain by Bonavia, Prado \& Barberá (2009). The DOCS scale was originally developed in English by Denison (1984), Denison \& Mishra (1995) and Fey \& Denison (2003). The Cognitive Interview was applied to a sample of 15 individuals with supervisory or managerial positions in organizations with 25 or more employees. Based on the results of the interviews the

Tomás Vargas-Halabi, Universidad de Costa Rica, Costa Rica; Ronald Mora Esquivel, Instituto Tecnológico de Costa Rica, Costa Rica .

La correspondencia en relación con este artículo se dirige a Tomás Vargas-Halabi, Escuela de Psicología, Universidad de Costa Rica. E-mail: tomas.vargas@ucr.ac.cr
\end{abstract}


T. VARgas, R. Mora-Esquivel

scale was adjusted to local parlance. It was then applied in two samples, with similar characteristics, but belonging two different populations: a) 314 students drawn from 445 active students enrolled in Master's Programs (MBA and Project Management) at the Costa Rican Institute of Technology, whose prerequisite for admission stipulates experience in professional positions, preferably supervisory and managerial, and; b) 368 managers drawn from a sampling frame of 1743 companies from various business associations. Eight types of confirmatory factor analyses were performed using EQS 6.3 software in order to explore the dimensionality of the adapted scale and to debug the final version. The Cognitive Interview was confirmed as a valuable tool in this process and the final scale (denominated DOCSCR-V2) demonstrated adequate functioning in the Costa Rican organizational context.

Keywords: DOCS model, cognitive interview, scale adaptation, organizational culture, SEM, confirmatory factor analysis

\section{Introducción}

La medición desempeña un papel central para el desarrollo de cualquier disciplina científica (Bearden, Netemeyer, \& Haws, 2011; Crook, Ketchen, Combs, \& Todd, 2008; Roth, Schroeder, Huang, $\&$ Murat, 2008; Slavec \& Drnovšek, 2012). Métricas confiables y válidas son vitales para el avance de la teoría (Roth et al., 2008). Su elaboración permite crear mejores modelos explicativos al reducir la posibilidad de obtener resultados espurios.

La validez de las mediciones en Ciencias Sociales se puede entender como el grado en el que la teoría y las evidencias empíricas apoyan las interpretaciones que se hacen de las puntuaciones de una herramienta de medición (Santisteban, 2009). Si bien, el concepto de validez se considera hoy unitario (Martínez, Hernández, \& Hernández, 2006), la literatura refiere a varios procedimientos para recopilar evidencias de la misma, los cuales comúnmente se denominan tipos de validez -de contenido, de los procesos de respuesta, de la estructura interna del instrumento, convergente, discriminante y la relacionada con un criterio (Santisteban, 2009).

En la III Conferencia internacional denominada Advanced Seminar on Cognitive Aspects of Survey Methodology, celebrada en 1983, se llegó a un amplio consenso acerca de los componentes cognitivos involucrados en el proceso de respuesta a preguntas de instrumentos, tales como cuestionarios y escalas, a saber: a) comprensión del ítem, b) recuperación de la información, c) evaluación de la información y d) selección de la respuesta apropiada (Tourangeau \& Bradburn, 2010). De las anteriores, la comprensión es la etapa en la cual se presenta la mayor parte de las dificultades. Algunos de los problemas relacionados con esta fase son los siguientes: a) vocabulario difícil de entender, b) mal estilo de redacción, c) incorrecta comprensión del contenido de la pregunta, d) inadecuado orden de las preguntas y e) dificultad para comprender las instrucciones (Krosnick \& Presser, 2010).

Ahora bien, con el propósito de identificar y corregir los errores en el proceso de respuesta, la literatura recomienda realizar un pretest o prueba piloto del borrador del instrumento (Colton \& Covert, 2007; Iarossi, 2006; Martin, 2006). Entre la variedad de herramientas para este propósito se encuentra la Entrevista Cognitiva (EC), la cual ha venido siendo utilizada con mucha frecuencia (Beatty \& Willis, 2007; Blair \& Brick, 2010; DesRoches, 2009). Quizás, esto se debe a que la EC ofrece a los investigadores información para detectar y también corregir diversos problemas de los ítems. 
La EC es una metodología que puede ser utilizada para depurar o calibrar escalas no ajustadas a un contexto, especialmente, con respecto a los errores de comprensión. No detectar este tipo de problemas puede llevar a distorsionar lo que se desea medir (Vercellotti, 2008). En otras palabras, se aplicaría un mismo ítem con diferentes significados para cada persona. Ahora bien, una adecuada comprensión contribuye a la validez relacionada con los procesos de respuesta, porque facilita la apropiada ejecución del resto de tareas cognitivas implicados en responder a una pregunta.

En una reciente investigación, Vargas-Halabí y Mora-Esquivel (2016) realizaron un estudio para medir el efecto de rasgos de cultura organizacional (CO) en el desempeño innovador de empresas costarricenses. Para su estudio, seleccionaron la Denison Organizational Culture Survey (DOCS) desarrollada por Denison (1984), Denison \& Mishra (1995) y Fey \& Denison (2003) para medir la CO (véase Figura 1). Dichos autores justifican la elección de la DOCS debido a que: a) existe de una versión adaptada al castellano por Bonavia, Prado y Barberá (2009) con una muestra de la Universidad Politécnica de Valencia, España (en adelante DOCSEP); b) posee un amplio soporte empírico; c) evalúa dimensiones, no tipos de CO (Jung et al., 2009). Estos aspectos la vuelven particularmente útil para el contexto nacional; además, permite realizar evaluaciones cuantitativas (Denison, Neiminen \& Kotbra, 2012) por dimensiones, lo cual posibilita comparar organizaciones y establecer normas con referencia al mercado (Khan, 2014).

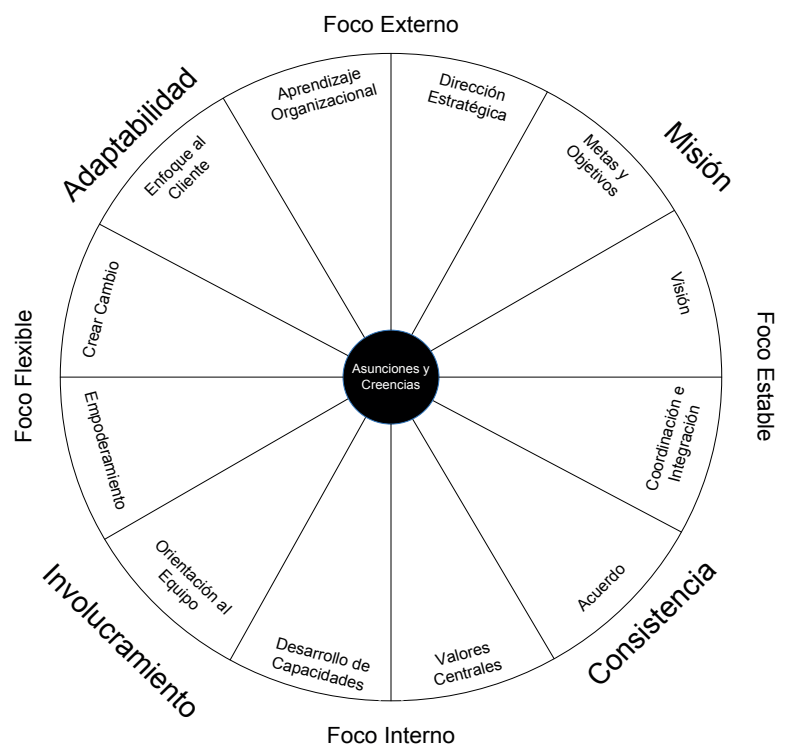

Figura 1. Modelo de CO propuesto por Denison y Mishra (1995).

Fuente. Elaboración propia

A lo largo de tres décadas, se han identificado en la literatura cuatro etapas del desarrollo de la DOCS (Vargas-Halabí, Mora-Esquivel y Ortíz, 2015). Primero, su diseño inicial; luego, se pasa a su consolidación y comprobación de evidencias de validez y confiabilidad. Seguidamente, su evaluación en contextos empresariales fuera de Estados Unidos y, finalmente, su uso para evaluar la relación entre CO y otros constructos (para más detalle, véanse, entre otros, Sharifirad y Ataei, 2012; Boyce, 
Niemen, Gillespie, Ryan y Denison, 2015, Denison, 1984; Denison \& Mishra, 1995; Denison, Haaland y Goelzer, 2003; Denison, Janovics, Young y Cho, 2006; Fey y Denison, 2003; Gillespie, Denison, Haaland, Smerek y Neale, 2008,).

El objetivo del presente artículo es presentar la experiencia y los resultados de utilizar de forma combinada la EC y el análisis factorial confirmatorio para adaptar la DOCSEP al contexto organizacional nacional con el propósito de brindar una herramienta útil, tanto a investigadores como practicantes en el área organizativa, que cuente con evidencias de validez en Costa Rica.

\section{Método}

En la Figura 2 se presenta un esquema que sintetiza la metodología del estudio, la cual contempló tres fases. En la primera se ejecutó una extensa revisión de la literatura para seleccionar una escala de $\mathrm{CO}$ adecuada para los objetivos de la investigación. Se procedió con detalle porque existe una gran diversidad de herramientas (Jung et al., 2009). Este análisis permitió seleccionar la DOCSEP debido a su soporte conceptual y empírico, su naturaleza dimensional y por encontrarse adaptada al castellano.

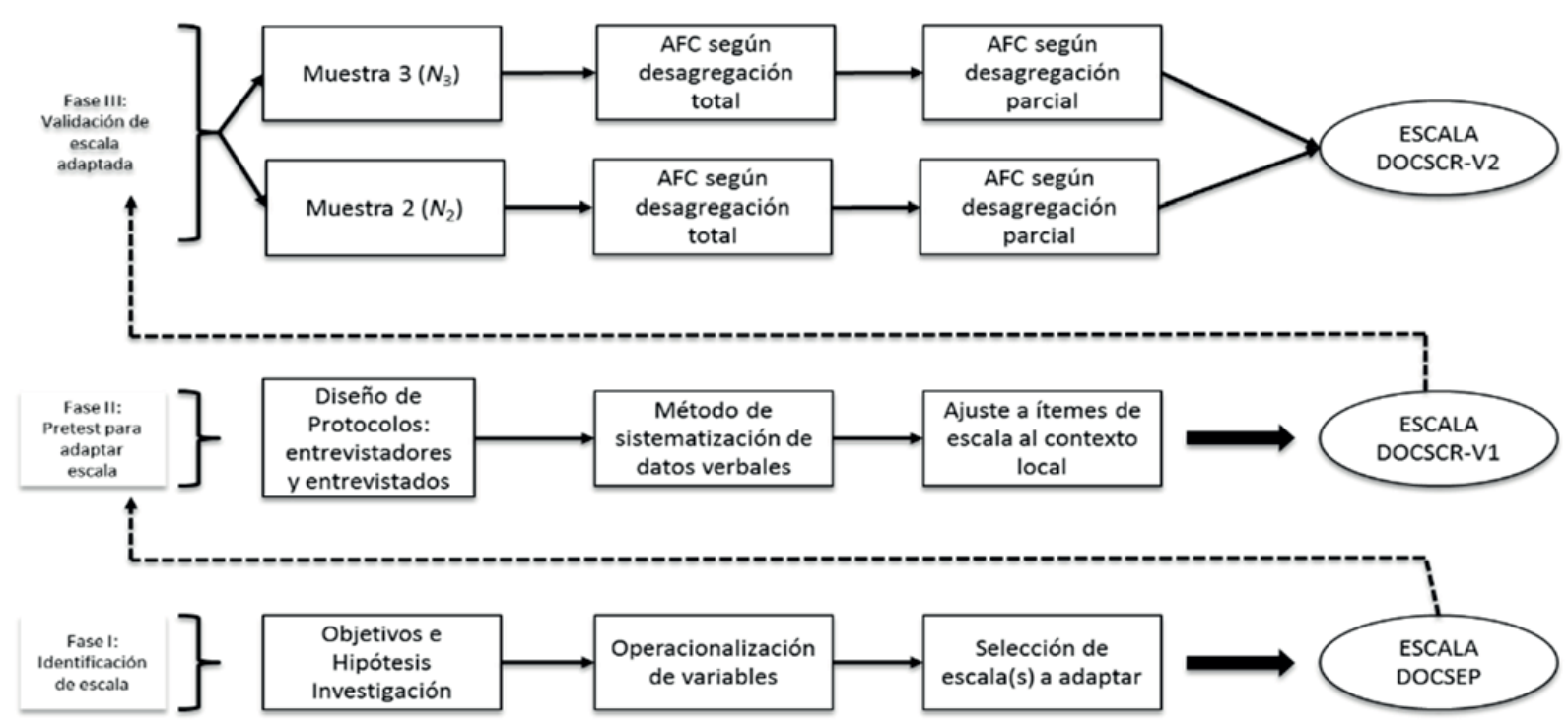

Figura 2. Diseño metodológico para adaptar la escala DOCSEP al contexto local.

Fuente. Elaboración propia.

En la segunda etapa se efectuó la adaptación de la DOCSEP al contexto organizacional costarricense mediante la aplicación de la EC, lo cual permitió desarrollar la DOCSCR-V1. En la tercera fase se efectuaron análisis factoriales confirmatorios para verificar que la dimensionalidad de la escala generada $\mathrm{y}$, con ello, los cambios propuestos con base en la EC, correspondían al modelo teórico original postulado por Denison (1984), Denison y Mishra (1995) y Fey \& Denison (2003). Así mismo, se evaluó el funcionamiento de cada ítem mediante la valoración de sus saturaciones. 


\section{Participantes}

Es importante recordar que el propósito del estudio fue adaptar y ofrecer evidencias de la validez acerca de la escala DOCSEP en el contexto organizacional costarricense. La naturaleza de los procedimientos para obtener tales evidencias requieren estrategias metodológicas diferentes. Por tal razón, el diseño de investigación se considera mixto, específicamente exploratorio secuencial (Creswell, 2014). Así, en el caso de la muestra $1\left(N_{1}\right)$ se utilizó una aproximación cualitativa y en las muestras $2\left(N_{2}\right)$ y 3 $\left(N_{3}\right)$ una cuantitativa.

En el caso de la muestra 1 se utilizó un muestreo dirigido, característico de los enfoques cualitativos, en el que son seleccionadas de una manera deliberada lugares o personas, con el fin de obtener los datos más completos y relevantes para comprender algún aspecto importante de la investigación (Yin, 2011). Para este estudio en particular, interesaron los procesos cognitivos implicados en la respuesta a los ítems de la escala DOCSEP. De acuerdo con la tipología mencionada por Creswell (2013), el muestreo fue dirigido mediante criterios, los cuales aseguraron la calidad de la información. Se seleccionaron personas capaces de reportar información verbalmente durante la entrevista cognitiva (EC), además de poseer características parecidas a los participantes de las muertes 1 y 2. Ello con el fin de obtener información del funcionamiento de los ítems con personas con características similares a las que finalmente responderían a la escala DOCSCR-V1.

La $N_{1}$ estuvo conformada por 15 participantes con características similares a las de los sujetos de la muestras $N_{2}$ y $N_{3}$. A cada persona de $N_{1}$ se le aplicó una EC acerca de 20 preguntas de la DOCSEP. De esta forma, se evitaron los efectos de la fatiga y se logró que cada ítem fuese evaluado por 5 personas, lo cual se encuentra dentro del rango mencionado por Smith-Castro y Molina (2011). También, se consideró el principio de saturación de información para determinar el momento para suspender el muestreo. Los participantes de esta muestra fueron mayoritariamente hombres $(60 \%)$, con puestos de supervisión o gerencia en organizaciones de los sectores de servicio, manufactura y comercio. Todos pertenecían a empresas con más de 25 empleados. Tenían, al menos, un año de laborar en la organización y contaban con formación universitaria.

En el caso de la $N_{2}$, tal como se mencionó, el enfoque fue cuantitativo. La población de referencia fueron 445 estudiantes activos de los programas de Maestría en Administración de Empresas y Gestión de Proyectos del Instituto Tecnológico de Costa Rica durante el primer semestre del 2014. La admisión a estos posgrados requiere experiencia laboral en cargos profesionales, preferiblemente a nivel de supervisión o gerencia, lo cual aseguró informantes con conocimientos de la dinámica y entorno de la organización en la que trabajaban.

Se utilizó un muestreo no probabilístico mediante el cual se alcanzó un 70 \% de la población (314 estudiantes). A los participantes que conformaron la $N_{2}$ se les aplicó la DOCSCR-V1 (véase ítemes en el apéndice 3$)$. En cuanto a la composición de la muestra, la mayoría fueron hombres (70 \%) con formación profesional en ingenierías (61\%). El promedio de años de laborar en la empresa fue 5.49 con una $D E=$ 4.34. Trabajaban, principalmente, en compañías de los sectores de servicios (65\%), manufactura (23\%) y, en menor medida, del sector comercial (12\%). La mayoría de los sujetos procedían de empresas con más de 100 colaboradores $(75 \%)$, consideradas como grandes dentro del contexto organizacional local. 
A los participantes de la $N_{3}$ también se les aplicó la DOCSCR-V1. Esta muestra fue seleccionada de manera no probabilística y estuvo conformada por 368 gerentes provenientes de una población de 1743 empresas con el fin de asegurar variabilidad entre las organizaciones. Para ello, se utilizó información de diversas asociaciones empresariales costarricenses (por ejemplo, PROCOMER, CADEXCO, CAMTIC, CACIA, entre otras). En lo referente a las características de los informantes, se observó una distribución equitativa entre hombres y mujeres. En su mayoría contaban con formación profesional a nivel de Bachillerato, Licenciatura (65\%) y Maestría (22\%) o de Técnicos (13\%), de las áreas de ciencias económicas (56 \%) y de ingenierías (18\%). La media de años de laborar para la empresa fue 7.8 con una $D E=7.8$. Es de destacar que se obtuvo un balance entre Pymes (53\%) y empresas grandes (48 \%). Lo mismo que por sector de actividad, específicamente: manufactura (40\%), servicios (30\%) y comercio $(30 \%)$.

Si bien es cierto, tanto $N_{2}$ como $N_{3}$ constituyen muestras no probabilísticas, se consideraron los criterios sugeridos por Highhouse y Gillespie (2009) para garantizar la calidad. Ahora bien, tomando en cuenta la naturaleza de las muestras y para dar mayor sustento a los resultados, se decidió realizar una replicación de los análisis cuantitativos en muestras de poblaciones diferentes.

En cuanto al tamaño de $N_{2}$ y $N_{3}$, es importante evidenciar que la DOCSEP permitió determinar, a priori, el modelo factorial confirmatorio de la escala. Por ello, se procedió a aplicar el procedimiento propuesto por MacCallum, Browne y Sugawara (1996) para determinar el número de casos con base en la potencia del Error de Aproximación Media Cuadrática (RMSEA, por sus siglas en inglés). Para esto, se estableció un ajuste aceptable de acuerdo con Lee, Cai y MacCallum (2012) (.05 $\leq R M S E A \leq .08)$. También, se especificaron 1362 grados de libertad $(g l)$ correspondientes a la DOCSEP, así como error Tipo I $(\alpha)$ y la Potencia Estadística (1- $\beta$ ) usuales (.05 y .80, respectivamente). Para efectuar el cálculo, se utilizó la herramienta de Preacher y Coffman (2006) que indicó 37 participantes. Valga acotar que la $N$ tan baja obedece a la gran cantidad de $g l$. Por esta razón, se recurrió a otra metodología, en este caso la desarrollada por Westland (2015), para lo cual se mantuvo los valores del $\alpha$ y $1-\beta$, con 16 factores latentes (correspondientes a la DOCSEP) y un umbral mínimo de .30 para los coeficientes a detectar en el modelo (tamaño del efecto). Para efectuar el cálculo, se utilizó la herramienta desarrollada por Soper (2016) que arrojó 116 casos. Como queda en evidencia, $N_{2}$ y $N_{3}$ superan el tamaño mínimo de participantes de acuerdo con la metodología de Westland (2015).

\section{Instrumentos}

Se utilizó la adaptación al castellano de la DOCS realizada por Bonavia et al. (2009) -que hemos denominado DOCSEP. Consta de 60 ítems, agrupados en 12 subdimensiones y 4 dimensiones. Estos son evaluados de acuerdo con una escala tipo likert de 5 puntos. Para aplicar la EC, se desarrolló un protocolo para la entrevistadora (véase apéndice 1) y 3 para los informantes. Estos últimos con 20 preguntas de las diferentes dimensiones de la DOCSEP (véase Apéndice 2) con el fin de poder controlar cualquier posible sesgo y los efectos de la fatiga.

Una vez realizados los ajustes a la DOCSEP, con base en los resultados de la EC, se le denominó DOCSCR-V1 y fue aplicada a los participantes de la $N_{2}$ y $N_{3}$. La versión final de la herramienta se denominó DOCSCR-V2, versión Vargas-Halabi \& Mora-Esquivel (2016) (véase Apéndice 3). Siguiendo lo 
recomendado por Krosnick y Presser (2010), se mantuvo la escala likert de endosamiento, pero se aumentó a 7 intervalos con 3 anclas, a saber: 1=En desacuerdo; 4= Ni de acuerdo ni en desacuerdo; 7= De acuerdo.

\section{Procedimiento}

Para la aplicación de la EC se siguieron las recomendaciones de Smith-Castro y Molina (2011). Se utilizó el procedimiento de pensar en voz alta ("think aloud") en el que se le solicita a los entrevistados verbalizar lo que piensan mientras responden a las preguntas (Beatty \& Willis, 2007).

La entrevistadora fue una estudiante avanzada del Bachillerato en Psicología de la Universidad de Costa Rica con formación técnica en Dirección de Recursos Humanos. Se efectuaron varios ensayos de conducta hasta que la entrevistadora alcanzó un nivel de manejo adecuado de los protocolos. Durante el entrenamiento se hizo énfasis en recopilar información relativa a los aspectos sugeridos por Tran (2009), a saber: a) claridad, b) conveniencia de la pregunta en términos de la cultura y los valores de las personas, c) dificultad y d. relevancia. Esto por cuanto, en cualquier estudio, es fundamental que las personas que respondan a un cuestionario o escalas comprendan la pregunta en el mismo sentido que lo hacen los investigadores (Tourangeau \& Bradburn, 2010). Asimismo, se instruyó a la entrevistadora en indagar acerca de cualquier otro aspecto que considerara relevante del proceso de respuesta a los ítems.

La primera aplicación se efectuó, en conjunto, con un investigador para asegurar que se estuviese ejecutando de forma adecuada. El resto de aplicaciones las realizó la entrevistadora sola. Todas las entrevistas, previa autorización del informante, fueron grabadas para ser transcritas en un archivo en Word. Para iniciar el análisis de los datos textuales, los investigadores leyeron varias veces cada entrevista. A continuación, marcaron en amarillo los segmentos de textos con observaciones, recomendaciones o comentarios acerca del funcionamiento de los ítems. Dichos segmentos se "cortaron" y "pegaron" en un nuevo archivo que contenía todas las observaciones por ítem. Estos resultados fueron sintetizados en un formato similar al utilizado por Hunter y DeMaio (2003) (véase ejemplo en la Tabla 1).

\section{Tabla 1}

Ejemplo de formato para la sistematización de los datos textuales de la EC utilizada en los items de la DOCSEP que presentaban aspecto para mejorar

\begin{tabular}{ll}
\hline Número de Ítem en DOCSEP & 22 \\
Protocolo & A \\
Código del ítem & A9 \\
Número de entrevistados & 5 \\
Contenido del ítem original & "Este grupo tiene una cultura «fuerte»". \\
Preocupaciones & $\begin{array}{l}\text { la palabra "fuerte" no es considerada por los participantes como adecuada. } \\
\text { Recomendaciones }\end{array}$ \\
$\begin{array}{l}\text { los entrevistados sugieren utilizar la frase "propia de la organización" o "constante". } \\
\text { Los investigadores evalúan las propuestas; sin embargo, consideran que ambas } \\
\text { opciones desdibujan el propósito del ítem, por lo que se recomienda buscar una } \\
\text { redacción alternativa. }\end{array}$ \\
$\begin{array}{l}\text { Redactar el ítem de la siguiente manera "Este grupo tiene una cultura organizacional } \\
\text { distintiva". }\end{array}$
\end{tabular}

Fuente. Elaboración propia. 
Las observaciones se analizaron desde una perspectiva sintáctica; es decir, con referencia a las reglas para la correcta utilización de las palabras y oraciones. También, se incluyó el plano semántico que se refiere al significado del lenguaje (Baggini \& Fosl, 2010). En síntesis, se evaluaron principalmente aspectos relativos a la adecuada escritura y compresión de los ítems. Para complementar el análisis de los datos textuales con frecuencias, se seleccionaron los códigos de los apartados I y II del protocolo propuesto por Presser y Blair (1994), los cuales hacen referencia a las dificultades sintácticas y semánticas de los reactivos. Con base en esta información, se realizaron las modificaciones que los investigadores consideraron apropiadas dando origen a la DOCSCR-V1 de 60 ítems.

Seguidamente, se procedió a aplicar la DOCSCR-V1 a la $N_{2}$. Esta fue realizada por estudiantes avanzados de la carrera de Psicología bajo un estricto protocolo y supervisión de un profesional con experiencia. En caso de la $N_{3}$ se realizaron, al menos, tres llamadas a los contactos de todas las organizaciones del marco muestral. También, se efectuó un seguimiento de, al menos, tres llamadas para obtener el cuestionario. En todos los casos, se garantizó el anonimato del informante y de la empresa. Valga señalar que, en ambos casos, se efectuó una cuidadosa aplicación del instrumento.

Con la información de ambas muestras se procedió a efectuar ocho tipos de análisis factoriales confirmatorios. Estos se ejecutaron en secuencia; es decir, primero los de la $N_{2}$ y luego los de la $N_{3}$. Igualmente, se realizaron en el orden que se describen con el fin de no sesgar los análisis. El primer análisis factorial confirmatorio (AFC) se efectuó con los 60 reactivos, con el fin de evaluar el funcionamiento de cada ítem y la estructura de la escala, aunque este enfoque disminuye las posibilidades de obtener un buen ajuste en el caso de instrumentos con varias dimensiones (Williams, Vandenberg, \& Edwards, 2009).

El segundo se realizó de acuerdo con lo sugerido por Possakoff, MacKenzie, Lee y Podsakoff (2013), para lo cual se añadió un factor latente exógeno para cargar los ítems con saturaciones bajas, con el propósito de verificar si compartían algún tipo de varianza no contemplada por el modelo conceptual. El tercer AFC se realizó excluyendo los ítems con saturaciones muy bajas, lo cual dio origen a la DOCSCR-V2 de 53 reactivos (véase apéndice 3). En el cuarto se utilizaron parcelas y se empleó como indicador el puntaje promedio de cada uno de los subfactores de la DOCSCR-V2 (doce en total, tres por cada dimensión). Según Little, Rhemtulla, Gibson y Schoemann (2013), el uso de parcelas puede considerarse como cualquier otra herramienta de medición que, en este caso, tiene como potencial brindar resultados más interpretables. Además, permite mejorar la relación del número de casos por parámetro (Little et al., 2002), lo cual es importante para el posterior uso del instrumento con Métodos de Ecuaciones Estructurales (SEM), porque se trata de una técnica que demanda muestras grandes (Kline, 2011; Schumacker \& Lomax, 2010; Ullman, 2013). Los análisis fueron realizados en el software EQS 6.3 que emplea el modelo Bentler-Weeks (Bentler, 2006). Este brinda la posibilidad de calcular estadísticos robustos utilizando máxima verosimilitud cuando se incumple el supuesto de normalidad multivariada (Byrne, 2006).

\section{Resultados}

\section{Adaptación de la DOCSEP: Entrevista cognitiva}

Como se puede observar en la Tabla 2 se modificaron 20 de los 60 ítems que conforman la DOCSEP (un $33.3 \%$ de sus reactivos). Las modificaciones se agruparon, principalmente, en ajustes de 
vocabulario con el fin de delimitar mejor los aspectos que incluye y excluye el ítem. En el ámbito de comprensión, un primer punto por señalar es que en 6 ítems los entrevistados manifestaron la necesidad de eliminar y sustituir términos para darles un sentido más acorde con el contexto local. A continuación, algunos ejemplos: a) la palabra "banquillo" en el ítem 12 evocó un sentido deportivo, lo cual se eliminó; b) en el ítem 16 la palabra "pregonan" suscitó el comentario en la mayoría de entrevistados de que la redacción no fue hecha para nuestro contexto, por lo tanto se sugirió que fuese sustituida por la palabra "predican con el ejemplo"; c) la palabra "de continuo", en el ítem 33, provocó que varios entrevistados manifestaran dificultad en el proceso de lectura y comprensión del reactivo, por lo que propusieron sustituirla por "continuamente".

\section{Tabla 2}

Comparación entre items originales de la DOCSEP y los de la DOCSCR-V1 como resultado del pretest

\begin{tabular}{|c|c|c|c|}
\hline $\begin{array}{l}\text { Número de } \\
\text { Ítem }\end{array}$ & $\begin{array}{c}\text { Ítem original } \\
\text { DOCSEP (España) }\end{array}$ & $\begin{array}{c}\text { Decisión acorde } \\
\text { con observaciones y } \\
\text { sugerencias en la EC }\end{array}$ & $\begin{array}{c}\text { Ítem adaptado para usarlo en la versión } \\
\text { DOCSCR-V1 (contexto local) }\end{array}$ \\
\hline 2 & $\begin{array}{l}\text { "Las decisiones } \\
\text { con frecuencia se } \\
\text { toman en el nivel que } \\
\text { dispone de la mejor } \\
\text { información". }\end{array}$ & Agregar una palabra. & $\begin{array}{l}\text { "Las decisiones con frecuencia se toman } \\
\text { en el nivel jerárquico que dispone de la } \\
\text { mejor información". }\end{array}$ \\
\hline 7 & $\begin{array}{l}\text { "Trabajar en este } \\
\text { grupo es como formar } \\
\text { parte de un equipo". }\end{array}$ & Eliminar una palabra. & $\begin{array}{c}\text { "Trabajar en este grupo es formar parte } \\
\text { de un equipo". }\end{array}$ \\
\hline 8 & $\begin{array}{l}\text { “Acostumbramos a } \\
\text { realizar las tareas en } \\
\text { equipo, en vez de } \\
\text { descargar el peso en la } \\
\text { dirección". }\end{array}$ & $\begin{array}{l}\text { Reemplazar una palabra } \\
\text { por otra palabra. }\end{array}$ & $\begin{array}{c}\text { "Acostumbramos a realizar las tareas en } \\
\text { equipo, en vez de descargar el peso en la } \\
\text { gerencia". }\end{array}$ \\
\hline 9 & $\begin{array}{l}\text { "Los grupos y no los } \\
\text { individuos son los } \\
\text { principales pilares de } \\
\text { esta organización". }\end{array}$ & $\begin{array}{l}\text { Reemplazar cuatro } \\
\text { palabras por otras tres. }\end{array}$ & $\begin{array}{l}\text { "Los grupos y no los individuos son la } \\
\text { base de esta organización". }\end{array}$ \\
\hline 11 & $\begin{array}{c}\text { “La autoridad se } \\
\text { delega de modo que } \\
\text { las personas puedan } \\
\text { actuar por sí mismas". }\end{array}$ & $\begin{array}{l}\text { Reemplazar cinco palabras } \\
\text { por otras ocho. }\end{array}$ & $\begin{array}{c}\text { "La autoridad se delega de modo que } \\
\text { las personas posean autonomía en el } \\
\text { desarrollo de sus funciones". }\end{array}$ \\
\hline
\end{tabular}

Continúa... 
T. Vargas, R. Mora-Esquivel

\begin{tabular}{|c|c|c|c|}
\hline 12 & $\begin{array}{l}\text { "Las capacidades } \\
\text { del "banquillo" (los } \\
\text { futuros líderes en el } \\
\text { grupo) se mejoran } \\
\text { constantemente" }\end{array}$ & Eliminar cinco palabras. & $\begin{array}{l}\text { "Las capacidades de los futuros líderes } \\
\text { se mejoran constantemente". }\end{array}$ \\
\hline 13 & $\begin{array}{l}\text { "Este grupo invierte } \\
\text { continuamente en } \\
\text { el desarrollo de las } \\
\text { capacidades de sus } \\
\text { miembros". }\end{array}$ & $\begin{array}{l}\text { Eliminar una palabra y } \\
\text { reemplazar dos palabras } \\
\text { por otras dos. }\end{array}$ & $\begin{array}{c}\text { "La organización invierte en el } \\
\text { desarrollo de las capacidades de sus } \\
\text { miembros". }\end{array}$ \\
\hline 16 & $\begin{array}{l}\text { "Los líderes y } \\
\text { directores practican lo } \\
\text { que pregonan". }\end{array}$ & $\begin{array}{l}\text { Reemplazar cuatro } \\
\text { palabras por otras cuatro. }\end{array}$ & $\begin{array}{c}\text { "Los líderes y directores predican con el } \\
\text { ejemplo". }\end{array}$ \\
\hline 17 & $\begin{array}{l}\text { "Existe un estilo } \\
\text { de dirección } \\
\text { característico con un } \\
\text { conjunto de prácticas } \\
\text { distintivas". }\end{array}$ & $\begin{array}{l}\text { Reemplazar cinco palabras } \\
\text { por otras seis. }\end{array}$ & $\begin{array}{l}\text { "Existe un estilo de dirección conocido } \\
\text { por todos y con características que lo } \\
\text { distinguen". }\end{array}$ \\
\hline 19 & $\begin{array}{l}\text { "Ignorar los valores } \\
\text { esenciales de este } \\
\text { grupo te ocasionará } \\
\text { problemas". }\end{array}$ & $\begin{array}{l}\text { Reemplazar dos palabras } \\
\text { por otras dos. }\end{array}$ & $\begin{array}{l}\text { "Ignorar los valores esenciales de este } \\
\text { grupo te traerá consecuencias". }\end{array}$ \\
\hline 22 & $\begin{array}{l}\text { "Este grupo tiene una } \\
\text { cultura «fuerte»". }\end{array}$ & $\begin{array}{l}\text { Reemplazar una palabra } \\
\text { por otras dos. }\end{array}$ & $\begin{array}{l}\text { "Este grupo tiene una cultura } \\
\text { organizacional distintiva". }\end{array}$ \\
\hline 26 & $\begin{array}{l}\text { "Nuestra manera de } \\
\text { trabajar es consistente } \\
\text { y predecible". }\end{array}$ & Eliminar dos palabras. & $\begin{array}{l}\text { "Nuestra manera de trabajar es } \\
\text { consistente". }\end{array}$ \\
\hline 32 & $\begin{array}{l}\text { "Respondemos bien } \\
\text { a los cambios del } \\
\text { entorno". }\end{array}$ & $\begin{array}{l}\text { Eliminar una palabra y } \\
\text { reemplazar dos palabras } \\
\text { por otras tres. }\end{array}$ & $\begin{array}{l}\text { "Respondemos bien a los cambios } \\
\text { propuestos por el mercado". }\end{array}$ \\
\hline 33 & $\begin{array}{l}\text { "Adoptamos de } \\
\text { continuo nuevas y } \\
\text { mejores formas de } \\
\text { hacer las cosas". }\end{array}$ & $\begin{array}{l}\text { Reemplazar dos palabras } \\
\text { por otra palabra. }\end{array}$ & $\begin{array}{l}\text { "Adoptamos continuamente nuevas y } \\
\text { mejores formas de hacer las cosas". }\end{array}$ \\
\hline 36 & $\begin{array}{l}\text { "Los comentarios } \\
\text { y recomendaciones } \\
\text { de nuestros clientes } \\
\text { conducen a menudo a } \\
\text { introducir cambios". }\end{array}$ & $\begin{array}{l}\text { Reemplazar dos palabras } \\
\text { por otras tres palabras. }\end{array}$ & $\begin{array}{l}\text { "Los comentarios y recomendaciones } \\
\text { de nuestros clientes conducen a nuestra } \\
\text { organización a introducir cambios". }\end{array}$ \\
\hline
\end{tabular}

Continúa... 
ADAPTACIÓN Y DIMENSIONALIDAD DE LA ESCALA DOCS EN EL CONTEXTO ORGANIZACIONAL COSTARRICENSE

\section{9}

41

53

42
"Nuestras decisiones ignoran con frecuencia los intereses de los clientes".

"Consideramos el fracaso como una oportunidad para aprender y mejorar".

"Tomar riesgos
e innovar son
fomentados y
recompensados".

"Nos aseguramos que

"la mano derecha sepa lo que está haciendo la izquierda".
Reemplazar tres palabras por otras tres palabras.

"Nuestras decisiones toman en cuenta los intereses de los clientes".

Reemplazar dos palabras por otras tres palabras.

Eliminar cuatro palabras y modificar redacción en dos palabras.

Eliminar diez palabras e incorporar nueve palabras
Reemplazar una palabra por otra

\author{
“Consideramos los errores cometidos \\ como oportunidades para aprender y \\ mejorar". \\ "Innovar es fomentado y \\ recompensado".
}

"Nos aseguramos de que todas las personas conozcan el trabajo de otros colaboradores".
"La gerencia nos conduce hacia los
objetivos que tratamos de alcanzar". objetivos que tratamos de alcanzar".

Fuente. Elaboración propia.

En segundo término, tres ítems requirieron una modificación sustancial en la estructura de la redacción con el fin de facilitar la comprensión y conservar el contenido teórico. Se trató de los ítems 17, 42 y 45. En el caso del primero, dos componentes en su redacción, "estilo de dirección característico" y "prácticas distintivas", provocaron duda en los entrevistados referente a lo que se estaba preguntando. En el ítem 42, la frase "tomar riesgos" se asoció con realizar decisiones de manera precipitada o con poca información, lo cual le dio una connotación negativa. En el último, la frase "que la mano derecha sepa lo que está haciendo la izquierda" generó mucha confusión entre los informantes. En estos ítems, la entrevistadora solicitó la "traducción" o interpretación de cada reactivo por parte de los participantes. Pese a que los ítems eran difíciles de leer, su significado para los cinco entrevistados correspondió a lo esperado. Los cambios sugeridos pueden observarse en la Tabla 2.

Un tercer aspecto, que se observó en la EC, fue la connotación negativa para los participantes de los ítems 41, 42 y 39. Por ejemplo, en el primero, la palabra fracaso se sugirió cambiar por "error cometido". En cuarto lugar, en los ítems 2 y 36, los entrevistados manifestaron que frases del ítem provocaban ambigüedad y recomendaron modificarlas. Finalmente, en 11 ítems aconsejaron reemplazar términos para facilitar la lectura $(7,9,11,12,19,22,26,32,33,39$ y 41). La Tabla 2 permite observar más en detalle cada uno de los cambios comentados anteriormente.

Un aspecto que generó dificultad, en la mayoría de los entrevistados, fue la definición del marco temporal para la escala. Al respecto, los participantes sugirieron "el último año". En esta dirección, es 
importante apuntar que una de las estrategias para mejorar la recuperación de la información es hacer lo más corto posible el período de referencia de la respuesta (Krosnick \& Presser, 2010). En este caso, para definir el marco temporal, se tomó en consideración que la CO involucra fenómenos organizativos que se vinculan con las operaciones, internas y externas, de las empresas. Asimismo, muchas de las actividades implicadas en sus rasgos se ejecutan anualmente debido a los ciclos propios del ecosistema social en el que opera la empresa, por lo que resulta razonable plantear el "último año" como marco temporal de referencia. Lo anterior resulta válido por cuando las empresas son sistemas abiertos; es decir, un conjunto interrelacionado de partes que realiza intercambios de recursos con el ambiente (Kast \& Rosenweing, 1972). Además, los aspectos intangibles también forman parte de los sistemas (Meadows \& Wright, 2008).

En términos generales, el procedimiento de codificación brindó un panorama global de los principales problemas de comprensión expresados por los entrevistados. Cabe señalar que, únicamente, en 2 de los doce indicadores de la DOCSEP (Dirección Estratégica y Visión) se mantuvieron sus ítems originales (sin corrección). El rango de modificaciones por indicador o subdimensión de la DOCSEP estuvo entre 1 y 3, lo cual denota uniformidad. En otras palabras, los cambios no se concentraron en un solo aspecto del instrumento. Los subíndices que más ítemes implicaron cambios fueron Desarrollo de capacidades y Trabajo en equipo (Dimensión Involucramiento), Valores centrales (Dimensión Consistencia) y Aprendizaje Organizacional (Dimensión Adaptabilidad) (véase tabla 3).

\section{Tabla 3}

Ajustes realizados a la DOCSEP codificadas de acuerdo con algunos criterios del protocolo de Presser y Blair (1994)

\begin{tabular}{|c|c|c|c|c|c|c|}
\hline Índices & $\begin{array}{l}\text { 1. Exceso } \\
\text { de texto }\end{array}$ & $\begin{array}{l}\text { 2. Mala } \\
\text { estructura }\end{array}$ & $\begin{array}{l}\text { 3. Límites } \\
\text { poco claros }\end{array}$ & $\begin{array}{l}\text { 4.Términos no } \\
\text { comprendidos }\end{array}$ & $\begin{array}{l}\text { 5. Dos tópicos en } \\
\text { una pregunta }\end{array}$ & $\begin{array}{l}\text { Frecuencia } \\
\text { por línea }\end{array}$ \\
\hline Empoderamiento & & & 2 & 2 & & 1 \\
\hline Trabajo en equipo & & & 7 & 8,9 & & 3 \\
\hline Desarrollo de capacidades & 12 & & 11,13 & 12 & & 4 \\
\hline Valores centrales & & 17 & 19 & 16 & & 3 \\
\hline Acuerdo & & & 22 & & & 1 \\
\hline Coordinación e integración & & & & & 26 & 1 \\
\hline Orientación al cambio & & & 32 & 33 & & 2 \\
\hline Orientación al cliente & & 39 & 36 & & & 2 \\
\hline Aprendizaje organizacional & 42 & 42,45 & 41 & & & 4 \\
\hline \multicolumn{7}{|l|}{ Dirección estratégica } \\
\hline Metas y objetivos & & & & 53 & & 1 \\
\hline \multicolumn{7}{|l|}{ Visión } \\
\hline Frecuencia por columna & 2 & 4 & 9 & 6 & 1 & 22 \\
\hline
\end{tabular}

Nota. El número en cada celda indica el ítem modificado de la DOCS adaptada al castellano por Bonavia, Prado, Barberá (2009). Fuente. Elaboración propia. 


\section{Dimensionalidad de la escala: Análisis factoriales confirmatorios}

En general, Hair, Black, Babin, \& Anderson (2014) sugieren el uso de Chi-cuadrado $\left(\chi^{2}\right)$, Índice de Ajuste Comparativo (CFI, por sus siglas en inglés) y RMSEA para evaluar los modelos SEM. En el primer caso se ha demostrado que es un estadístico muy sensible al tamaño de la N (Schumacker \& Lomax, 2010; Stevens, 2009); es decir, con muchos casos tiende a ser estadísticamente significativo, lo que lleva a rechazar la $\mathrm{H} 0$ que indica que el modelo ajusta. Dado el tamaño de $N_{2}$ y $N_{3}$, se tuvo cautela con la significancia de Satorra-Bentler Scaled Chi-Square $\left(S B \chi^{2}\right)$. Con respecto al CFI, el valor mínimo para un adecuado ajuste se fijó en .90 (Keith, 2015), aunque algunos autores sugieren como ideal 95 (Baubeta, Mallou, Piñeiro, \& Mangin, 2006; Rencher \& Christensen, 2012). Para el RMSEA, puntajes por debajo de .05 indican un buen ajuste mientras que valores mayores a .08 reflejan un mal funcionamiento del modelo (Bentler, 2006; Schumacker \& Lomax, 2010). En cuanto a las cargas, se estableció un umbral mínimo de $|.50|$ (Hair et al., 2014) o lo que es lo mismo un $25 \%$ de la varianza del ítem explicada por el factor latente. La consistencia interna se evaluó mediante el coeficiente de confiabilidad lineal para modelos SEM, denominado RHO (Yang \& Green, 2011), porque CO es un constructo multidimensional (Byrne, 2006).

Todos los análisis factoriales confirmatorios (AFC) fueron modelos de primer nivel; es decir, no se elaboraron modelos factoriales jerárquicos o de segundo nivel. Además, como era de esperar teóricamente, se observaron en 8 los modelos planteados correlaciones superiores a .70 entre las subdimensiones. Adicionalmente, en todos los análisis, el índice de mardía fue superior a 6 , lo que evidenció ausencia de normalidad multivariada (Álvarez, González, \& Mangin, 2006). Por esta razón, se utilizaron las versiones robustas de los tres índices mencionados, así como del Criterio de información de Akaike (AIC, por sus siglas en inglés) (Bentler, 2006). Este último permite comparar modelos con base en su ajuste y parsimonia. De acuerdo con Byrne (2006), el valor menor de AIC indica el mejor modelo de acuerdo con los dos aspectos indicados. Sin embargo, este índice acarrea una penalización a menores g1 (Byrne, 2006).

El primer modelo se ejecutó con los datos correspondientes a la DOCSCR-V1 de los participantes de la $N_{2}$. Como puede observarse en la en la Tabla 4, la $S B \chi^{2}$ fue estadísticamente significativa, $p<.001$, por lo cual se rechazó la H0 que establece que el modelo ajusta. Sin embargo, como se indicó, es probable que este resultado esté afectado por el número de casos. El CFI fue inferior al mínimo requerido, lo cual denotó mal ajuste. Pese a esto, el RMSEA indicó un apropiado funcionamiento (véase tabla 4). 
T. VARgas, R. Mora-Esquivel

Tabla 4

Índices y fiabilidad de los modelos

\begin{tabular}{|c|c|c|c|c|c|c|}
\hline$N$ & $\mathrm{AFC}$ & $S B \chi^{2 a}$ & $\mathrm{AIC}^{\mathrm{b}}$ & CFI & $\operatorname{RMSEA}_{(\alpha=10 \%)}$ & RHO \\
\hline \multirow{6}{*}{$N_{2}$} & 1. DOCSCR-V1, 60 ítems & $2671.16(1692 \mathrm{gl}) *$ & -712.84 & .87 & $.046,[.043 ; .050]$ & .97 \\
\hline & 2. DOCSCR-V1, factor latente & $2501.45(1685 \mathrm{gl})^{*}$ & -868.54 & .89 & $.042,[.038 ; .045]$ & \\
\hline & Redacción negativa & & & & & \\
\hline & 3. DOCSCR-V2, 53 ítems & 1990. $11(1307 \mathrm{gl})^{*}$ & -623.89 & .91 & $.043,[.039 ; .047]$ & .97 \\
\hline & 4. DOCSCR-V2, 12 parcelas & $72.52(48 \mathrm{gl}) *$ & -23.48 & .99 & $.040,[.019 ; .059]$ & .96 \\
\hline & 5. DOCSCR-V1, 60 ítems & $2533.25(1692 \mathrm{gl})^{*}$ & -850.75 & .88 & $.038,[.035 ; .041]$ & .97 \\
\hline \multirow{4}{*}{$N_{3}$} & 6. DOCSCR-V1, factor latente & $2307.44(1685 \mathrm{gl})^{*}$ & -1062.56 & .91 & $.033,[.029 ; .036]$ & \\
\hline & Redacción negativa & & & & & \\
\hline & 7. DOCSCR-V2, 53 ítems & $1832.23(13 \mathrm{gl})^{*}$ & -781.77 & .92 & $.034,[.030 ; .037]$ & .98 \\
\hline & 8. DOCSCR-V2, 12 parcelas & $106.99(48 \mathrm{gl}) *$ & 10.98 & .97 & $.059,[.044 ; .074]$ & .97 \\
\hline
\end{tabular}

Nota a $S B \chi^{2}$ corregida de acuerdo con Bentler (2006). ${ }^{\text {b }}$ Por no ser modelos anidados, se utilizó el AIC robusto que brinda el

EQS. El valor menor indica mejor ajuste entre un conjunto de modelos (Byrne, 2006).

$* \mathrm{p}<.001$. Fuente. Elaboración propia

Con respecto a las cargas factoriales, la gran mayoría fueron estadísticamente significativas, $p<$ .05 , con valores muy por encima de $|.50|$ (véase apéndice 3). Empero, se identificaron 6 reactivos con saturaciones por debajo de |.20|, específicamente: 15, 24, 29, 34, 43 y 58. Como puede observarse, en el apéndice 3, todos estos ítems poseen una redacción inversa. Valga señalar que el ítem 54 también cuenta con este tipo de redacción, pero su saturación fue de .44 , estadísticamente significativa, $p<.05$.

Estos resultados sugieren que la redacción negativa constituyó una fuente de varianza común que explica más de los ítems invertidos que las dimensiones de la DOCSCR-V1. Esto quedó demostrado con el modelo 2, que añade el factor latente, denominado Redacción inversa, para el cual también se cargaron los ítems negativos. En este segundo análisis, se observaron varios aspectos muy relevantes. En primer lugar, los índices de ajuste mostraron una mejoría consistente -aunque todavía insuficiente- al añadir el constructo latente. En segundo término, el AIC robusto fue menor que el del modelo 1 (véase Tabla 4). En tercer lugar, los ítems con redacción negativa mostraron saturaciones estadísticamente significativas, $p<$ .05 , y de magnitudes superiores (en un rango entre .37 y .57) con el factor Redacción inversa, en comparación con las que se obtuvieron con su respectiva subdimensión de la DOCSCR-V1 (véase Apéndice 3).

Estos resultados brindaron soporte suficiente para eliminar los ítems redactados en forma inversa. Además, afortunadamente, todos cargaban a subfactores diferentes con lo cual ninguno quedó con menos de 4 indicadores. Al respecto, como se observa en la tabla 5, el modelo 3 con la escala DOCSCR-V2 de 53 ítems mostró índices de ajuste adecuados y una alta confiabilidad. Empero, el AIC robusto fue mayor que el modelo 1, lo cual puede deberse a la diferencia en gl (385). Aun así, se prefiere este modelo 3 al modelo 1 dado su mejor ajuste y adecuadas saturaciones, las cuales estuvieron en un rango |.49|.a|.93|(véase Apéndice 3). En virtud de estos resultados, en el AFC 5, se utilizaron parcelas, lo cual mejoró aún más el ajuste global de la escala propuesta como lo evidencia el CFI y el RMSEA robustos; asimismo, las saturaciones 
no bajaron de .72. No obstante, su AIC robusto fue el mayor de todos los modelos. Esto puede explicarse en términos de que las parcelas reducen los gl del modelo drásticamente. Sin embargo, la utilización de parcelas puede ser una opción recomendable para investigadores que deseen utilizar la DOCSCR-V2 en conjunto con otros instrumentos y requieran mejorar la relación de casos por parámetros estimados.

Ahora bien, con el fin de bridar mayor soporte a la escala propuesta se realizaron los mismos análisis factoriales con la $N_{3}$. Como se observa en la tabla 5, se replicaron los resultados a nivel de índices de ajuste y fiabilidad de la escala. De nuevo, la inclusión del factor latente Redacción inversa mejoró el funcionamiento del modelo. En esta dirección, los ítems con redacción negativa volvieron a presentar saturaciones muy superiores con el factor Redacción inversa que con su respectiva subdimensión de la DOCSCR-V1. Adicionalmente, la eliminación de estos reactivos permitió alcanzar un ajuste adecuado para la escala propuesta (DOCSCR-V2), pero, de nuevo con el menor AIC robusto. Por su parte, el modelo con parcelas de la $N_{3}$ mostró aún un mejor desempeño que en la $N_{2}$ (véase tabla 4), con cargas no menores a .83 (véase Apéndice 3).

Finalmente, es necesario resaltar que todos los reactivos modificados mostraron, en términos generales, sustracciones muy superiores al puntaje de corte (véase Apéndice 3). Esto, en conjunto con los resultados descritos previamente, sugiere que la escala DOCSCR-V2 logró adaptarse de manera que se ajustara a la dimensionalidad del modelo teórico.

\section{Discusión}

El propósito de este estudio fue presentar la eficacia de la EC como metodología para adaptar la escala DOCSEP al contexto organizacional costarricense. Al comparar los resultados, la DOCSCR-V2 mostró un mejor ajuste que la DOCSEP (CFI de .99 en la primera y .956 en la segunda; RMSEA de .040 en la primera y de .088 en la segunda). Adicionalmente, en la adaptación para Costa Rica se sometió a evaluación el modelo con los 60 ítems y se logró una mejora en la escala, en términos de ajuste del modelo y saturaciones, con la eliminación de 7 ítems. Más aún, cabe destacar que en este estudio se replicaron los resultados en dos muestras diferentes con resultados similares. En el caso de Bonavia et al. (2009), el estudio se realizó con una muestra que estuvo compuesta por 488 personas de diferentes departamentos de investigación y desarrollo (docentes, investigadores y becarios) de la Universidad Politécnica de Valencia.

Los resultados del ajuste obtenido para Costa Rica son similares a los reportados por Denison, Neiminen \& Kotbra (2012). Estos autores, entre los años 1997 y 2001, aplicaron la DOCS a una muestra de 35.474 individuos de 160 compañías, de gran tamaño. El modelo totalmente desagregado obutvo un CFI de .98 y un RMSEA de .05. En el presente estudio se logró un buen ajuste de la escala DOCSCR-V2 con un CFI no tan alto $(\mathrm{N} 2=.91 ; \mathrm{N} 3=.92)$, pero con un RMSEA de mejor nivel $(\mathrm{N} 2=.042 ; \mathrm{N} 3=.034)$. En este sentido, puede afirmarse que los resultados reportados no presentan diferencias notables. Por tal razón, resulta razonable considerar que los ítems mejorados para Costa Rica mediante la EC contribuyeron a que se mantuvieran adecuadamente las dimensiones que son la base del modelo teórico de la DOCS. Lo anterior subraya la importancia de trabajar la primera etapa del proceso de respuesta al ítem (comprensión).

Hay que reconocer una limitación, en el sentido de la que la EC no detectó el mal funcionamiento de los ítems con redacción inversa, lo cual constituye una línea de investigación por desarrollar en cuanto 
T. VARgas, R. MORA-Esquivel

a la aplicación de la EC. Pese a esto, una contribución de los análisis cuantitativos de este estudio es que claramente indican que la redacción negativa es una fuente de varianza muy importante y que este tipo de ítems tienden a no funcionar de manera adecuada. De hecho, la literatura más reciente indica que es preferible no utilizar este tipo de escritura (Krosnick \& Presser, 2010). Más aún, en Costa Rica, Solís (2015) demuestra empíricamente que los ítems negativos afectan el funcionamiento y la consistencia interna de las escalas, lo cual refuerza nuestro hallazgo.

A la luz de los resultados descritos, se puede argumentar que la EC es una herramienta muy útil para adaptar las herramientas de medición. Consideramos que coadyuva no solo a la validez relacionada con los procesos de respuesta, sino también a la estructura interna de los instrumentos. Otro elemento que resultó de mucho valor, para darle más solidez al estudio, fue replicar los resultados en muestras diferentes. Tómese también, en consideración, que se debió calcular una cantidad grande de parámetros. Por ejemplo, el modelo 1 (de la N2) y el modelo 5 (de la N3) calculan 138 parámetros, por lo que es factible que aún, con un $95 \%$ de confianza, algunos parámetros varían por azar. De aquí la importancia de replicar.

El hecho de haber logrado adaptar el instrumento de manera que se apegara a la estructura teórica del modelo y que, además, se haya replicado en dos muestras independientes constituye un paso importante en la validación de la DOCS para el contexto local. No obstante, es necesario continuar los estudios, principalmente, para evaluar la validez convergente, discriminante y relacionada con un criterio del instrumento, lo cual constituyen líneas futuras de investigación. Una vez realizados estos estudios, se estaría en posibilidad de desarrollar normas con respecto al mercado (e.g. percentiles). Lo anterior podría convertirse en un punto de referencia para las empresas que permitan realizar auditorías internas con respecto a $\mathrm{CO}$ e incluso en su relación con otros constructos de interés para el contexto organizacional.

Finalmente, el modelo de medida ajustó tanto en su forma desagregada como en su versión con parcelas. En este sentido, el modelo de parcelas está respaldado por un buen ajuste del modelo desagregado. En otras palabras, la escala de 53 ítems funciona de forma adecuada; por consiguiente, el ajuste del modelo con parcelas no es espurio. Esto brinda confianza a los investigadores de que la escala funciona de manera razonable y que es factible recurrir al uso de parcelas con modelos estructurales complejos, con el fin de reducir las demandas de SEM en lo relativo al tamaño de la muestra.

\section{Referencias}

Álvarez, N., González, J., \& Mangin, J. (2006). Normalidad y otros supuestos en el análisis de covarianzas. En J. Lévy Mangin \& J. Varela Mallou (Eds.), Modelización con estructuras de covarianza en ciencias sociales: Temas esenciales, avanzados y aportaciones especiales (pp. 31-57). España: Netbiblo.

Sadegh Sharifirad, M., \& Ataei, V. (2012). Organizational culture and innovation culture: exploring the relationships between constructs. Leadership \& Organization Development Journal, 33(5), 494-517.

Baggini, J., \& Fosl, P. (2010). The philosopher's toolkit: A compendium of philosophical concepts and methods. Oxford: Wiley-Blackwell.

Baubeta, A., Mallou, J., Piñeiro, J., \& Mangin, J. (2006). El análisis factoral confirmatorio. En J. Lévy Mangin \& J. Varela Mallou (Eds.), Modelización con estructuras de covarianza en ciencias sociales: Temas esenciales, avanzados y aportaciones especiales (pp. 119-143). España: Netbiblo. 
Bearden, W., Netemeyer, R., \& Haws, K. (2011). Handbook of marketing scales. Multi-Item measures for marketing and consumer behavior research. California: SAGE.

Beatty, P., \& Willis, G. (2007). Research synthesis: The practice of cognitive interviewing . Public Opinion Quaterly, 71(2), 287-311.

Bentler, P. (2006). EQS 6 Structural Equations Program Manual. California: Multivariate Software, Inc.

Blair, J., \& Brick, P. D. (2010). Methods for the analysis of cognitive interviews. Proceedings of the Section on Survey Research Methods, 1-2, 3739-3748.

Bonavia, T., Prado, G. V., \& Barberá, T. D. (2009). Adaptación al castellano y estructura factorial del Denison Organizational Culture Survey. Psicothema, 21(4), 633-638.

Boyce, A., Niemen, L., Gillespie, M., Ryan, A., \& Denison, D. (2015). Which comes first, organizational culture or performance? A longitudinal study of causal priority with automobile dealerships. Journal of Orgnizational Behavior, 36(3), 339-359. doi:10.1002/job.1985

Byrne, B. (2006). Structural equations modeling with EQS: Basics concepts, applications, and programming. New York: Routledge.

Colton, D., \& Covert, R. (2007). Designing and constructing instruments for social research and evaluation. California: John Wiley \& Sons, Inc.

Creswell, J. (2013). Qualitative Inquiry and Reseach Desing: Choosing Among Five Approaches (Third, ed.) California: Sage

Creswell, J. W. (2014). Research design: Qualitative, quantitative, and mixed methods approaches. (Fourth, ed.). California: Sage publications

Crook, R., Ketchen, D., Combs, J., \& Todd, S. (2008). Strategic resources and performance: A Meta-analysis. Strategic Management Journal, 29, 1141-1154. doi:10.1002/smj

Denison, D. (1984). Bringing corporate culture to the bottom line. Organizational Dynamics, 13(2), 4-22.

Denison, D., \& Mishra, A. (1995). Toward a theory of organizational culture and effectiveness. Organization Science, 6(2), 201-223.

Denison, D., Haaland, S., \& Goelzer, P. (2003). Corporate culture and organizational efectiveness: is there a similar pattern around the world? En W. Mobley, \& P. Dorfman, Advances in gobal leadership (Vol. 3, págs. 205-226). Bradford: Emerald Group Publishing Ltd.

Denison, D., Janovics, J., Young, J., \& Cho, H. (2006). Diagnosing organizational cultures: validating a model and method. Lausanne: International Institute for Management Development.

Denison, D., Neiminen, L., \& Kotbra, L. (2012). Diagnosing organizational culture: An conceptual and empirical review of effectiveness surveys. European Journal of Work and Organizational Psycology, 23(1), 1-17.

DesRoches, D. (2009). Establishment survey. En P. Lavrakas, Encyclopedia of survey research methods (pp. 239-243). California: SAGE. 
T. VARGas, R. Mora-EsQuivel

Fey, C., \& Denison, D. (2003). Organizational culture and efectiveness: Can american theory be applied in Rusia? Organization Science, 14(6), 686-706.

Gillespie, M., Denison, D., Haaland, S., Smerek, R., \& Neale, W. (2008). Linking organizational culture and customer satisfaction: Results from two companies in different industries. European Journal of Work and Organizational Psychology, 17(1), 112-132.

Hair, J., Black, W., Babin, A., \& Anderson, R. (2014). Multivariate Data Analysis: Pearson new international edition (Setima ed.). New Jersey, Essex: Pearson.

Highhouse, S., \& Gillespie, J. (2009). Do Samples Really Matter That Much? En C. Lance, \& R. Vandenberg, Statistical and Methodological Myths: Doctrine, Verity and Fable in the Organizational and Social Sciences (pp. 247-265). New York: Routledge.

Hunter, J., \& DeMaio, T. (2003). Results \& Recommendations from the Cognitive Pretesting of the 2003 Public School Questionnaire from the Schools and Staffing Survey. Washington D.C.: Statistical Research Division U.S. Bureau of the Census.

Iarossi, G. (2006). The power of survey desing: A user 's guide for managing survey, interpreting result, and influencing respondents. Washington, DC: World Bank .

Jung, T., Scott, T., Davies, H., Bower, P., Whalley, D., McNally, R., \& Mannion, R. (November-December de 2009). Instruments for exploring organizational culture: A review of the literature. Public Administration Review, 69(6), 1087-1096.

Kast, F., \& Rosenweing, J. (1972). General system theory: Applications for organizations and management. Academy of Management Journals, 15(4), 447- 465.

Keith, T. (2015). Multiple regression and beyond: An introduction to multiple regression and structural equation modeling. New York: Routledge.

Khan, S. (2014). Scholarly contributions of Daniel Denison in the field of learning organizations. International Journal of Organizational Learning and Change, 2(1), 6-10.

Kline, R.(2011). Principles and practice of structural equation modeling.(Third ed.). New York: The Guilford Press.

Krosnick, J.A., \& Presser, S. (2010). Question and questionnaire design. In P. Mardsen, \& J. Wright(Eds.), Handbook of survey research (Second ed., pp. 263-314). United Kingdom: Emerald Group Publishing Limited.

Lee, T., Cai, L., \& MacCallum, R. (2012). Power analysis for tests of Structural Equation Models. En R. Hoyle, Handbook of structural equation modeling (pp. 181-194). New York : The Guilford Press.

Little, T., Cunningham, W., Shahar, G., \& Widaman, K. (2002). To parcel or not to parcel: Exploring the question, weighing the merits. Structural Equation Modeling, 9(2), 151-173.

Little, T., Rhemtulla, M., Gibson, K., \& Schoemann, A.M. (2015). Why the items versus parcels controversy needn't be one. Psychological Methods, 28(3), 285-300. DOI: 10.1037/a0033266

MacCallum, R., Browne, M., \& Sugawara, H. (1996). Power analysis and determination of sample size for covariance structure modeling. Psychological Methods, 1(2), 130-149. 
ADAPTACIÓN Y DIMENSIONALIDAD DE LA ESCALA DOCS EN EL CONTEXTO ORGANIZACIONAL COSTARRICENSE

Martin, E. (2006). Survey Questionnaire Construction. In M. E, Survey Questionnaire Construction (pp. 1-12). Washington: Director's Office U.S. Census Bureau.

Martínez, M., Hernández, M., \& Hernández, M. (2006). Psicometría. Madrid: Alianza Editorial, S.A.

Meadows, D. H., \& Wright, D. (2008). Thinking in Systems: A primer. Chelsea: Green Publishing.

Possakoff, P., MacKenzie, S., Lee, J., \& Podsakoff, N. (2013). Common method biases in behavioral research: A critical review of literature and recommended remedies. Journal of Applied Psycholoy, 88(1), 879-903.

Preacher, K. J., \& Coffman, D. L. (Mayo de 2006). Computing power and minimum sample size for RMSEA [Computer software]. Retrieved from http://www.quantpsy.org/rmsea/rmsea.htm

Presser, S., \& Blair, J. (1994). Survey pretesting: Do different methods produce different results?. Sociological methodology, 24, 73-104. DOI: 10.2307/270979.

Rencher, A., \& Christensen, W. (2012). Methods of multivariate analysis. New Jersey: John Wiley \& Sons, Inc.

Roth, A., Schroeder, R., Huang, X., \& Murat, M. (2008). Handbook of Metrics for research in operations Management: Multi-item Measurement scales and objetive items. California: SAGE.

Solís, M. (2015). The dilemma of combining positive and negative items in scales. Psicothema, 27(2), 192-200.

Santisteban, C. (2009). Principios de pscicometría. Madrid: Sïntesis.

Schumacker, E., \& Lomax, R. (2010). A beginner's guide to structural equation modeling. New York: Routledge Taylor \& Francis Group.

Slavec, A., \& Drnovšek, M. (2012). A perspective on scale development in entrepreneurship research. Economic and Business Review, 14(19), 39-62.

Smith-Castro, V., \& Molina, M. (2011). La entrevista cognitiva: Guía para su aplicación en la evaluación y mejoramiento de instrumentos de papel y lápiz. San José, Costa Rica: Instituto de Investigaciones Psicológicas, Universidad de Costa Rica.

Soper, Daniel. (2015). A-priori Sample Size Calculator for Structural Equation Models. [Programa de Cómputo]. Retrieved from www.danielsoper.com/statcalc

Stevens, J. (2009). Applied multivariate statistics for the social sciences (Fifth ed.). New Jersey: Routledge.

Tourangeau, R., \& Bradburn, N.M. (2010). The Psychology of Survey Response. In P. Mardsen , \& J. Wright (Eds.), Handbook of survey research (Seconded., pp. 263-314). United Kingdom: Emerald Group Publishing Limited.

Tran, T. (2009). Developing Cross-Cultural Measurement. New York: Oxford University Press, Inc.

Ullman, J. (2013). Structural equation modeling. In B. Tabachnick, \& L. Fidell (Eds.), Using Multivariate Statistics (pp. 676-780). Boston: Pearson.

Vargas-Halabí, T., \& Mora-Esquivel, R. (2016). Efecto de la cultura organizativa en la innovacion: Un estudio empírico. Revista de Ciencias Económicas, 2(34), 9-46. 
T. VARGas, R. Mora-EsQuivel

Vargas-Halabí, T., Mora-Esquivel, R. \& Ortiz, C. (2015). Cultura organizativa e innovación: un análisis temático en empresas de Costa Rica. Revista Tec Empresarial, 9(2), 7-18.

Vercellotti, T. (2008). Comprehension. In P. Lavrakas, Encyclopedia of survey research methods (pp. 117-118). California: SAGE.

Westland, Christopher. (2015). Structural Equation Models: From paths to networks. New York: Springer.

Williams, L., Vandenberg, R., \& Edwards, J. (2009). Structural equation modeling in management research: A guide for improved analysis. The Academy of Management Annals, 3(1), 543-604.

Yang, Y., \& Green, S. B. (2011). Coefficient alpha: A reliability coefficient for the 21 st century?. Journal of Psychoeducational Assessment, 29(4), 377-392.

Yin, R. (2011). Qualitative research from start to finish. New York: The Guilford Press.

Recibido: 15 de diciembre de 2016 Revisión recibida: 27 de Julio de 2017

Aceptado: 10 de Agosto de 2017

\section{Sobre los autores:}

Tomás Vargas-Halabi es bachiller y licenciado en Psicología por la Universidad de Costa Rica, máster en Administración de Empresas por el Instituto Tecnológico de Costa Rica (ITCR) y doctor en Dirección de Empresas por la Universidad de Valencia, España. Actualmente, funge como profesor catedrático de la Escuela de Psicología donde imparte cursos de Psicobiología, Psicología Organizacional y Metodologías de la Investigación. En investigación, sus líneas de trabajo se han enfocado en el campo organizacional con especial preferencia por un enfoque transdisplinario.

Ronald Mora Esquivel es bachiller y licenciado en Economía por la Universidad de Costa Rica, máster en Administración de Empresas por el Instituto Tecnológico de Costa Rica (ITCR) y doctor en Dirección de Empresas por la Universidad de Valencia, España. Actualmente, labora como docente e investigador del Centro de Investigaciones en Administración, Economía y Gestión Tecnológica en la Escuela de Administración de Empresas del ITCR. En investigación, sus líneas de trabajo se han enfocado a tópicos de finanzas, empresas de base tecnológica, innovación empresarial y otros relativos al recurso humano en la empresa (intraemprendedurismo y cultura organizativa). En docencia, labora para la Cátedra de Economía e imparte cursos para el Bachillerato y la Maestría. 


\section{Apendices}

\section{Apendice 1: Protocolo para el entrevistador}

\section{1-Consideraciones iniciales para el entrevistador}

Procurar un espacio adecuado (libre de distracciones para el desarrollo de la entrevista).

Saludo inicial.

Generar un ambiente de comodidad para el entrevistado.

Comentar la intención de la entrevista de manera informal.

Indicar las razones por las cuales se utilizará la grabadora y asegurar el acuerdo con el entrevistado.

\section{2-Instrucciones para el entrevistador}

\section{Contextualización}

Estamos realizando una investigación acerca de cómo personal de distintas empresas a nivel nacional interpretan los ítems que plantea el siguiente cuestionario.

\section{Consigna}

Para esto, le voy a entregar el cuestionario y le voy a pedir que lo llene como si estuviéramos realizando la evaluación. En esta etapa, nos interesa saber cómo está funcionando el cuestionario. Por eso, le voy a pedir que conforme lo va completando piense en voz alta. Es decir, que diga en voz alta todo lo que se le viene a la mente conforme lo va resolviendo. Es importante que tenga presente que lo que se quiere es adaptar el instrumento al contexto nacional, confirmar que el instrumento esté funcionando de manera adecuada, entonces no dude en decirme si algo le parece confuso y si algo se puede mejorar.

\section{Aclaración de otros aspectos}

Vamos a durar aproximadamente 60 minutos en todo el proceso. Antes de iniciar, ¿tiene alguna pregunta?

\section{Práctica}

Para irse acostumbrando a pensar en voz alta, vamos a practicar con un ejemplo: "Trate de visualizar su casa en este momento, empiece a caminar por su casa, por la sala, el comedor, la cocina, los cuartos, los baños... Ahora, por favor, dígame qué está viendo durante este recorrido. Ahora, indíqueme qué piensa mientras ve su casa".

\section{Grabación}

En este punto puede prender la grabadora. Indicar fecha y hora de la entrevista; entrevistador; puesto y profesión del entrevistado; área en la que trabaja. Cualquier otra información de carácter personal acerca del entrevistado o sensible de la empresa procure que no quede grabada. 
T. Vargas, R. Mora-Esquivel

\section{3-Inicio de las actividades}

Tener presente

Si el entrevistado manifiesta algún tipo de ansiedad (por ejemplo, siente que va a revelar información confidencial o delicada de la empresa) se debe disminuirla antes de entregar el protocolo. No mencionar aspectos de este tipo a menos de que sea un emergente propio del entrevistado.

\section{Entrega del protocolo}

\section{Instrucción de inicio}

Muy bien, ahora vamos a iniciar. Recuerde ir diciendo en voz alta todo lo que pase por su mente mientras lee y responde al ítem. Si no surge la información de manera espontánea, plantee las siguientes preguntas: ¿qué le pareció el ítem?; ¿el ítem le fue fácil o difícil de entender?; ¿es relevante en su contexto laboral y cultural?; ¿cómo llegó a la respuesta?

\section{Al finalizar}

Apagar la grabadora.

Dar las gracias.

Evacuar cualquier consulta. 


\section{Apendice 2}

\section{Instrucciones}

\section{Ejemplo de Protocolo A para el entrevistado (20 primeros ítems)}

A continuación, se le presentan una serie de frases relacionadas con cultura organizacional. Marque con una equis (X) del 1 al 5 cuán de acuerdo o en desacuerdo está con cada una de las frases. 1 es en Completo Desacuerdo y 5, en Completo Acuerdo.

\begin{tabular}{|c|c|c|c|c|c|c|}
\hline & & 1 & 2 & 3 & 4 & 5 \\
\hline A1 & $\begin{array}{l}\text { La mayoría de los miembros de este grupo están muy } \\
\text { comprometidos con su trabajo. }\end{array}$ & $(\quad)$ & $(\quad)$ & $(\quad)$ & $(\quad)$ & ( ) \\
\hline A5 & $\begin{array}{l}\text { La autoridad se delega de modo que las personas } \\
\text { puedan actuar por sí mismas. }\end{array}$ & $(\quad)$ & $(\quad)$ & $(\quad)$ & $(\quad)$ & $(\quad)$ \\
\hline A10 & Nuestra manera de trabajar es consistente y predecible. & $(\quad)$ & $(\quad)$ & $(\quad)$ & ( & ( ) \\
\hline A20 & $\begin{array}{l}\text { Tenemos una visión compartida de cómo será esta } \\
\text { organización en el futuro. }\end{array}$ & $(\quad)$ & $(\quad)$ & $(\quad)$ & $(\quad)$ & $(\quad)$ \\
\hline
\end{tabular}

\section{Ejemplo de Protocolo B para el entrevistado (20 segundos ítems)}

\section{Instrucciones}

A continuación, se le presenta una serie de frases relacionadas con cultura organizacional. Marque con una equis (X) del 1 al 5 cuán de acuerdo o en desacuerdo está con cada una de las frases. 1 es en Completo Desacuerdo y 5 , en Completo Acuerdo.

\begin{tabular}{|c|c|c|c|c|c|c|}
\hline & & 1 & 2 & 3 & 4 & 5 \\
\hline A2. & $\begin{array}{l}\text { Cada miembro cree que puede tener un impacto positivo } \\
\text { en el grupo. }\end{array}$ & $(\quad)$ & $(\quad)$ & $(\quad)$ & $(\quad)$ & $(\quad)$ \\
\hline A12. & $\begin{array}{l}\text { Los intentos de realizar cambios suelen encontrar } \\
\text { resistencias. }\end{array}$ & $(\quad)$ & $(\quad)$ & $(\quad)$ & $(\quad)$ & $(\quad)$ \\
\hline A16. & $\begin{array}{l}\text { Esta organización tiene una misión clara que le otorga } \\
\text { sentido y rumbo a nuestro trabajo. }\end{array}$ & $(\quad)$ & $(\quad)$ & $(\quad)$ & $(\quad)$ & $(\quad)$ \\
\hline A20. & $\begin{array}{l}\text { El pensamiento a corto plazo compromete a menudo } \\
\text { nuestra visión a largo plazo. }\end{array}$ & $(\quad)$ & $(\quad)$ & $(\quad)$ & $(\quad)$ & $(\quad)$ \\
\hline
\end{tabular}


T. VARGas, R. Mora-EsQuivel

\section{Ejemplo de Protocolo C para el entrevistado (20 terceros ítems)}

\section{Instrucciones}

A continuación, se le presentan una serie de frases relacionadas con cultura organizacional. Marque con una equis (X) del 1 al 5 cuán de acuerdo o en desacuerdo está con cada una de las frases. 1 es en Completo Desacuerdo y 5 , en Completo Acuerdo.

\begin{tabular}{|c|c|c|c|c|c|c|}
\hline & & 1 & 2 & 3 & 4 & 5 \\
\hline A4. & $\begin{array}{l}\text { La capacidad de las personas es vista como una fuente } \\
\text { importante de ventaja competitiva. }\end{array}$ & $(\quad)$ & $(\quad)$ & ( & $(\quad)$ & \\
\hline A9. & $\begin{array}{l}\text { Trabajar con alguien de otro grupo de esta organización } \\
\text { es como trabajar con alguien de otra organización. }\end{array}$ & $(\quad)$ & ( ) & ( & & \\
\hline A15. & $\begin{array}{l}\text { Nos aseguramos de que "la mano derecha sepa lo que } \\
\text { está haciendo la izquierda". }\end{array}$ & $(\quad)$ & ( ) & ( & & $(\quad)$ \\
\hline A20. & $\begin{array}{l}\text { Podemos satisfacer las demandas a corto plazo sin } \\
\text { comprometer nuestra visión a largo plazo. }\end{array}$ & $(\quad)$ & () & ( & $(\quad)$ & ( \\
\hline
\end{tabular}




\section{Apéndice 3}

Saturaciones de los ítems adaptados. Se incluyen en negrita los reactivos eliminados para conformar la escala DOCSCR-V2

\begin{tabular}{|c|c|c|c|c|c|c|c|c|c|c|}
\hline \multirow{2}{*}{ Subdimensiones/ítems } & \multicolumn{10}{|c|}{ Modelos } \\
\hline & 1 & & 2 & 3 & 4 & 5 & & 6 & 7 & 8 \\
\hline Empoderamiento $^{\text {b }}$ & & & & & $.82 * \mathrm{e}$ & & & & & $.85^{* \mathrm{e}}$ \\
\hline $\begin{array}{l}\text { 1. La mayoría de los miembros de este grupo están muy } \\
\text { comprometidos con su trabajo. }\end{array}$ & $.54 *$ & $.54 *$ & & $.54 *$ & & $.64 *$ & $.64 *$ & & $.64 *$ & \\
\hline $\begin{array}{l}\text { 2. Las decisiones, con frecuencia, se toman en el nivel } \\
\text { jerárquico que dispone de la mejor información }{ }^{\mathrm{a}} \text {. }\end{array}$ & $.50 *$ & $.50 *$ & & $.50 *$ & & $.54^{*}$ & $.54 *$ & & $.54 *$ & \\
\hline $\begin{array}{l}\text { 3. La información se comparte ampliamente y se puede } \\
\text { conseguir la información que se necesita. }\end{array}$ & $.71 *$ & $.71^{*}$ & & $.71 *$ & & $.68^{*}$ & $.68 *$ & & $.68^{*}$ & \\
\hline $\begin{array}{l}\text { 4. Cada miembro cree que puede tener un impacto } \\
\text { positivo en el grupo. }\end{array}$ & $.71 *$ & $.71^{*}$ & & $.70^{*}$ & & $.66^{*}$ & $.66^{*}$ & & $.66^{*}$ & \\
\hline $\begin{array}{l}\text { 5. La planificación de nuestro trabajo es continua e } \\
\text { implica a todo el mundo en algún grado. }\end{array}$ & $.67 *$ & $.67^{*}$ & & $.67 *$ & & $.67 *$ & $.67 *$ & & $.67 *$ & \\
\hline Orientación al equipo $^{\text {b }}$ & & & & & $.86^{* \mathrm{e}}$ & & & & & $.83 * \mathrm{e}$ \\
\hline $\begin{array}{l}\text { 6. Se fomenta activamente la cooperación entre los } \\
\text { diferentes grupos de esta organización. }\end{array}$ & $.76^{*}$ & $.76^{*}$ & & $.76^{*}$ & & $.77 *$ & $.77 *$ & & $.76^{*}$ & \\
\hline 7. Trabajar en este grupo es formar parte de un equipo ${ }^{a}$. & $.79 *$ & $.79 *$ & & $.78^{*}$ & & $.84^{*}$ & $.84 *$ & & $.84 *$ & \\
\hline $\begin{array}{l}\text { 8. Acostumbramos a realizar las tareas en equipo, en vez } \\
\text { de descargar el peso en la gerencia }{ }^{\text {a }} \text {. }\end{array}$ & $.69^{*}$ & $.70^{*}$ & & $.69^{*}$ & & $.72 *$ & $.72 *$ & & $.72 *$ & \\
\hline $\begin{array}{l}\text { 9. Los grupos y no los individuos son la base de esta } \\
\text { organización }^{\text {a. }} \text {. }\end{array}$ & $.66^{*}$ & $.67 *$ & & $.66^{*}$ & & $.53 *$ & $.53 *$ & & $.52 *$ & \\
\hline $\begin{array}{l}\text { 10. El trabajo se organiza de modo que cada persona } \\
\text { entiende la relación entre su trabajo y los objetivos de la } \\
\text { organización. }\end{array}$ & $.67 *$ & $.67^{*}$ & & $.67 *$ & & $.75^{*}$ & $.75^{*}$ & & $.75^{*}$ & \\
\hline Desarrollo de capacidades ${ }^{b}$ & & & & & $.81^{\mathrm{e}}$ & & & & & $.83^{\mathrm{e}}$ \\
\hline $\begin{array}{l}\text { 11. La autoridad se delega de modo que las personas } \\
\text { posean autonomía en el desarrollo de sus funciones }{ }^{\mathrm{a}} \text {. }\end{array}$ & $.57^{*}$ & $.57^{*}$ & & $.58 *$ & & $.57 *$ & $.57 *$ & & $.57 *$ & \\
\hline $\begin{array}{l}\text { 12. Las capacidades de los futuros líderes de la } \\
\text { organización se mejoran constantemente }{ }^{\text {a. }}\end{array}$ & $.78 *$ & $.78^{*}$ & & $.79 *$ & & $.77^{*}$ & $.77 *$ & & $.77 *$ & \\
\hline $\begin{array}{l}\text { 13. La organización invierte en el desarrollo de las } \\
\text { capacidades de sus miembros }{ }^{\mathrm{a}} \text {. }\end{array}$ & $.76^{*}$ & $.76^{*}$ & & $.79 *$ & & $.67 *$ & $.67 *$ & & .67 & \\
\hline $\begin{array}{l}\text { 14. La capacidad de las personas es vista como una } \\
\text { fuente importante de ventaja competitiva. }\end{array}$ & $.84 *$ & $.84 *$ & & $.84 *$ & & $.75^{*}$ & $.76^{*}$ & & $.76^{*}$ & \\
\hline $\begin{array}{l}\text { 15. A menudo, surgen problemas porque no disponemos } \\
\text { de las habilidades necesarias para hacer el trabajoc }\end{array}$ & -.01 & -.01 & $.37^{* d}$ & & & -.03 & -.03 & $.49^{* d}$ & & \\
\hline
\end{tabular}


T. Vargas, R. Mora-Esquivel

\begin{tabular}{|c|c|c|c|c|c|c|c|c|c|c|}
\hline Valores centrales $^{\text {b }}$ & & & & & $.77^{\mathrm{e}}$ & & & & & $.83^{\mathrm{e}}$ \\
\hline 16. Los líderes y directores predican con el ejemplo ${ }^{a}$. & $.72 *$ & $.72 *$ & & $.72 *$ & & $.72 *$ & $.72 *$ & & $.72 *$ & \\
\hline $\begin{array}{l}\text { 17. Existe un estilo de dirección conocido por todos y } \\
\text { con características que lo distinguena. }\end{array}$ & $.74 *$ & $.74 *$ & & $.73^{*}$ & & $.69^{*}$ & $.69 *$ & & $.69^{*}$ & \\
\hline $\begin{array}{l}18 \text { Existe un conjunto de valores claro y consistente que } \\
\text { rige la forma en que nos conducimos. }\end{array}$ & $.76^{*}$ & $.76^{*}$ & & $.76^{*}$ & & $.81^{*}$ & $.81 *$ & & $.82 *$ & \\
\hline $\begin{array}{l}\text { 19. Ignorar los valores esenciales de este grupo te traerá } \\
\text { consecuencias }{ }^{\mathrm{a}} \text {. }\end{array}$ & $-.49 *$ & $-.49 *$ & & $-.49 *$ & & $.57 *$ & $.57 *$ & & $.57 *$ & \\
\hline $\begin{array}{l}\text { 20. Existe un código ético que guía nuestro } \\
\text { comportamiento y nos ayuda a distinguir lo correcto. }\end{array}$ & $.56^{*}$ & $.56^{*}$ & & $.55^{*}$ & & $.61^{*}$ & .61 & & $.62 *$ & \\
\hline Acuerdo $^{b}$ & & & & & $.84^{\mathrm{e}}$ & & & & & $.87^{\mathrm{e}}$ \\
\hline $\begin{array}{l}\text { 21. Cuando existen desacuerdos, trabajamos intensamente } \\
\text { para encontrar soluciones donde todos ganen. }\end{array}$ & $.68 *$ & $.68^{*}$ & & $.68^{*}$ & & $.74^{*}$ & $.74 *$ & & $.75^{*}$ & \\
\hline $\begin{array}{l}\text { 22. Este grupo tiene una cultura organizacional } \\
\text { distintiva }{ }^{\text {a }}\end{array}$ & $.64 *$ & $.64 *$ & & $.63^{*}$ & & $.61^{*}$ & $.61 *$ & & $.62 *$ & \\
\hline $\begin{array}{l}\text { 23. Nos resulta fácil lograr el consenso, aun en temas } \\
\text { difíciles. }\end{array}$ & $.59 *$ & .59 & & $.60^{*}$ & & $.63^{*}$ & $.63 *$ & & $.63 *$ & \\
\hline $\begin{array}{l}\text { 24. A menudo, tenemos dificultades para alcanzar } \\
\text { acuerdos en temas clave }\end{array}$ & .14 & $.15^{*}$ & $.53^{* \mathrm{~d}}$ & & & $-.13 *$ & $-.13^{*}$ & $.48^{* d}$ & & \\
\hline $\begin{array}{l}\text { 25. Existe un claro acuerdo acerca de la forma correcta e } \\
\text { incorrecta de hacer las cosas. }\end{array}$ & $.70^{*}$ & $.70^{*}$ & & $.69^{*}$ & & $.73 *$ & $.73 *$ & & .74 & \\
\hline Coordinación e integración ${ }^{\mathrm{b}}$ & & & & & $.85^{\mathrm{e}}$ & & & & & $.85 *$ \\
\hline 26. Nuestra manera de trabajar es consistente ${ }^{\mathrm{a}}$. & $.69 *$ & $.69 *$ & & $.69 *$ & & $.68^{*}$ & $.68^{*}$ & & $.68^{*}$ & \\
\hline $\begin{array}{l}\text { 27. Las personas de diferentes grupos de esta } \\
\text { organización tienen una perspectiva común. }\end{array}$ & $.62 *$ & $.72 *$ & & $.72 *$ & & $.72 *$ & $.72 *$ & & $.73 *$ & \\
\hline $\begin{array}{l}\text { 28. Es sencillo coordinar proyectos entre los diferentes } \\
\text { grupos de esta organización. }\end{array}$ & $.64 *$ & $.64 *$ & & $.64^{*}$ & & $.66^{*}$ & $.66^{*}$ & & $.67 *$ & \\
\hline $\begin{array}{l}\text { 29. Trabajar con alguien de otro grupo de esta } \\
\text { organización es como trabajar con alguien de otra } \\
\text { organización*c }\end{array}$ & $.17 *$ & $.18^{*}$ & $.51^{* d}$ & & & -.10 & -.09 & $.44 * d$ & & \\
\hline $\begin{array}{l}\text { 30. Existe una buena alineación de objetivos entre los } \\
\text { diferentes niveles jerárquicos. }\end{array}$ & $.79 *$ & $.79 *$ & & $.79^{*}$ & & $.83 *$ & $.83 *$ & & $.83^{*}$ & \\
\hline Crear cambio $^{\text {b }}$ & & & & & $.84^{\mathrm{e}}$ & & & & & $.82 * \mathrm{e}$ \\
\hline $\begin{array}{l}\text { 31. La forma que tenemos de hacer las cosas es flexible y } \\
\text { fácil de cambiar. }\end{array}$ & $.73 *$ & $.73 *$ & & $.73^{*}$ & & $.57^{*}$ & $.57 *$ & & $.57 *$ & \\
\hline $\begin{array}{l}\text { 32. Respondemos bien a los cambios propuestos por el } \\
\text { mercado }^{\text {a }} \text {. }\end{array}$ & $.78^{*}$ & $.78^{*}$ & & $.78^{*}$ & & $.81^{*}$ & $.81 *$ & & $.82 *$ & \\
\hline
\end{tabular}


33. Adoptamos continuamente nuevas y mejores formas

de hacer las cosas ${ }^{\mathrm{a}}$.

34. Los intentos de realizar cambios suelen encontrar resistencias*c

35. Los diferentes grupos de esta organización cooperan, $\quad .67 * \quad .67 *$ a menudo, para introducir cambios.

Enfoque al cliente ${ }^{b}$

36. Los comentarios y recomendaciones de nuestros clientes conducen a nuestra organización a introducir cambios ${ }^{\mathrm{a}}$.

37. La información sobre nuestros clientes influye en nuestras decisiones. necesidades y deseos de nuestros clientes.

39. Nuestras decisiones toman en cuenta los intereses de $.85^{*} \quad .85 *$ los clientes ${ }^{\mathrm{a}}$.

40. Fomentamos el contacto directo de nuestra gente con $\quad .60 * \quad .60 *$ los clientes.

\section{Aprendizaje organizacional $^{b}$}

41. Consideramos los errores cometidos como oportunidades para aprender y mejorara ${ }^{\mathrm{a}}$.

42. Innovar es fomentado y recompensado .

43. Muchas ideas "se pierden por el camino"

44. El aprendizaje es un objetivo importante en nuestro trabajo cotidiano

45. Nos aseguramos de que todas las personas conozcan el trabajo de otros colaboradores ${ }^{\mathrm{a}}$.

\section{Dirección estratégica ${ }^{\mathrm{b}}$}

46. Esta organización tiene un proyecto y una orientación a largo plazo.

47. Nuestra estrategia sirve de ejemplo a otras organizaciones.

48. Esta organización tiene una misión clara que le otorga sentido y rumbo a nuestro trabajo.

49. Esta organización tiene una estrategia clara de cara al futuro.

$.75^{*} \quad .75^{*}$

$.74 * \quad .74 *$

$.68^{*} \quad .68 *$

$.75^{*} \quad .75^{*}$

$.70^{*} \quad .70 *$

$.72 * \quad .78 *$

$.80 * \quad .80 *$

$.88^{*} \quad .88 *$

$.94 * .93 *$
$.71 * \quad .71 *$

$.75^{*}$

$.75^{*}$

$.85 *$

$.60^{*}$

$.72^{\mathrm{e}}$

$.84 * \quad .84 *$

$.83 *$

$-.14 \quad-.13$

$.56^{* \mathrm{~d}}$

$67 *$

$.71 * \quad .71 *$

$.71 *$

$.70^{*}$

$.78^{*} .78^{*}$

$.78^{*}$

$.71 * \quad .71 *$

$.71 *$

$.80 * \quad .80 *$

$.80 *$

$.81 * \quad .81 *$

$.81 *$

$.66^{*} \quad .66^{*}$

$.66^{*}$

$.83^{\mathrm{e}}$

$.84 *$ e

$.71 *$

$.68^{*}$

$.70 * \quad .70 *$

$.66^{*} \quad .66^{*}$

$.67 *$

$.75^{*}$

$\begin{array}{lll}-.21 * & -.20 * \quad .53 * \mathrm{~d}\end{array}$

$.20 * \quad .21 * \quad .50 * \mathrm{~d}$

$.71 *$

$.77 * \quad .77 *$

$.78^{*}$

$.66^{*}$

$.65^{*} .65^{*}$

$.66^{*}$

$.86^{\mathrm{e}}$

$.87^{\mathrm{e}}$

$.79 *$

$.71 *$

.79

$.79 *$

.

$.80 *$

$.68^{*} \quad .68^{*}$

$.68^{*}$

$.88^{*}$

$.90 * \quad .90 *$

$.90 *$

$.93 *$

$.89 * \quad .89 *$

$.89 *$ 
T. Vargas, R. Mora-Esquivel

50. La orientación estratégica de esta organización no me
resulta clarac.

Metas y objetivos ${ }^{b}$

51. Existe un amplio acuerdo sobre las metas por conseguir.

52. Los líderes y directores fijan metas ambiciosas pero $\quad .88 * \quad .88 *$ realistas.

53. La gerencia nos conduce hacia los objetivos que tratamos de alcanzara

54. Comparamos continuamente nuestro progreso con los $\quad .83 * \quad .83 *$ objetivos fijados.

55. Las personas de esta organización comprenden lo que . $77 * \quad .77 *$ hay que hacer para tener éxito a largo plazo.

Visión

56. Tenemos una visión compartida de cómo será esta organización en el futuro.

57. Los líderes y gerentes tienen una perspectiva a largo $\quad .81 * \quad .81 *$ plazo.

58. El pensamiento a corto plazo compromete a menudo $\quad .20 * \quad .20 * \quad .45 * \mathrm{~d}$ nuestra visión a largo plazo $^{\mathrm{c}}$.

59. Nuestra visión genera entusiasmo y motivación entre nosotros.

60. Podemos satisfacer las demandas a corto plazo $\sin \quad .70 * \quad .70 *$ comprometer nuestra visión a largo plazo.

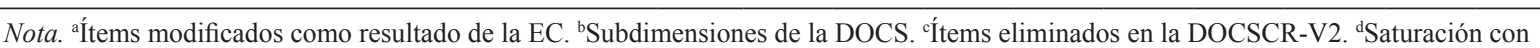
el factor latente Redacción inversa. eSaturación con la subdimensión tomada como parcela.

$* \mathrm{p}<.05$
$-.26 * \quad-.24 * \quad .55^{\mathrm{d} *}$

$.86^{\mathrm{e}}$

$.94 * \mathrm{e}$

$\begin{array}{llll}.78 * & .82 * & .82 * & .81 * \\ .88 * & .86 * & .86 * & .85 * \\ .84 * & .80 * & .80 & .80 * \\ .82 * & & & \\ & .86 * & .86 & .85 * \\ .77 * & & & .83 *\end{array}$

$.90^{\mathrm{e}}$

$.90 * \mathrm{e}$

$\begin{array}{llll}.84 * & .80 * & .80 * & .80 *\end{array}$

$\begin{array}{llll}.81 * & .79 * & .79 * & .79 *\end{array}$

$\begin{array}{lll}-.02 & -.00 \quad .51 * \mathrm{~d}\end{array}$

$.78 *$

$.83 * .83 *$

$.83 *$

$.70 *$

$.66 \quad .66$

$.66 *$ 